\title{
Le Dictionnaire de l'Académie Française : un modèle lexicographique en évolution
}

\author{
Rey, Christophe \\ Université de Picardie Jules Verne - LESCLaP (CERCLL) \\ christophe.rey@u-picardie.fr
}

\section{Introduction}

La longévité exceptionnelle du Dictionnaire de l'Académie Française, répertoire qui en est aujourd'hui à sa neuvième édition et qui a parcouru pas moins de cinq siècles, ne s'explique pas seulement par la sécurité de publication que lui assure son label de « dictionnaire de l'institution ». Très souvent stigmatisé pour la lenteur de son élaboration, l'ouvrage des Académiciens est doté d'une pérennité qui s'explique selon nous également par le fait qu'il s'agit d'un dictionnaire qui a su évoluer au fil du temps, intégrant à travers son lexique les évolutions linguistiques ${ }^{1}$ et culturelles ${ }^{2}$ les plus significatives. L'ouvrage de Bernard Quemada et Jean Pruvost (1997) consacré aux préfaces des huit premières édition de ce dictionnaire constitue à ce titre une référence incontournable pour mesurer ce constat ${ }^{3}$.

Dans cette communication, nous souhaitons revenir sur cette image du Dictionnaire de l'Académie Française (désormais $D A F$ ) en tant que modèle lexicographique sans cesse en évolution. Nous nous inscrivons donc à ce titre dans la lignée de la publication récente d'un numéro de la revue Études de Linguistique Appliquée (ÉLA) ${ }^{4}$ consacré à l'ouvrage.

En rebondissant sur certaines analyses récentes (REY, C., PIEROZAK, (À paraître), REY, C., (2012a), SOUFFI, 2009), nous chercherons à montrer ici que cette neuvième livraison du répertoire - toujours en cours de rédaction - présente à travers plusieurs choix lexicographiques majeurs que nous allons détailler un visage considérablement renouvelé.

\section{De 1986 à aujourd'hui, une neuvième édition en voie de finalisation}

La neuvième et dernière édition du $D A F$ se situe dans le sillage d'une longue tradition lexicographique initiée au XVII ${ }^{\mathrm{e}}$ siècle et ponctuée par des publications à chaque siècle, une au XVII ${ }^{\mathrm{e}}$ (1694), quatre au $\mathrm{XVIII}^{\mathrm{e}}(1718,1740,1762,1798)$, deux au XIX ${ }^{\mathrm{e}}(1835,1878)$ et une au $\mathrm{XX}^{\mathrm{e}}(1932-35)$. La dernière édition du $D A F$ est publiée presque trois cents ans après la première édition. Si nous exceptons justement cette première édition dont la rédaction particulièrement lente - de 1637 à 1694 - a été vivement critiquée à maintes reprises (Furetière, 1685), cette dernière édition est aussi celle qui paraît avec le plus d'écart avec l'édition qui la précède.

Ébauchée durant le mandat de Secrétaire perpétuel de l'Académie Française de Maurice Druon, la neuvième édition du $D A F$ sera pour la première fois publiée sous la forme de fascicules à partir de 1986, puis sous la forme de deux tomes successivement édités chez Fayard en 1992 (articles A à Enzyme) et 2000 (articles Éocène à Mappemonde). Non encore achevée ${ }^{5}$, cette édition continue de paraître de manière apériodique sous la forme de fascicules dans la liste des Documents administratifs du Journal officiel ${ }^{6}$.

\section{Des innovations}

À l'image de l'apport scientifique de chacune de ses éditions précédentes (Quemada, 1997), le DAF offre à travers cette neuvième édition un répertoire original, livrant une vision nouvelle de la langue française. 
L'originalité de cette ultime édition se manifeste non seulement à travers l'accroissement particulièrement conséquent de sa nomenclature, mais aussi et surtout à travers l'introduction de nombreuses « innovations » lexicographiques dont les plus significatives ${ }^{7}$ vont succinctement être décrites ci-dessous.

\title{
3.1 Remarques normatives
}

$\mathrm{Au}$ rang des premières innovations importantes que propose la neuvième édition du $D A F$, figure tout d'abord le mécanisme d'introduction de « remarques normatives »:

\begin{abstract}
«Le Dictionnaire de l'Académie française, à côté des indications concernant la graphie, la prononciation, le pluriel des mots, la conjugaison des verbes, etc., qui sont nécessaires dans tout dictionnaire de langue, insère dans certains articles des remarques destinées à attirer l'attention sur des modes, des habitudes plus ou moins répandues dans la langue courante et contraires au bon usage. » (Site internet de l'Académie française)
\end{abstract}

Destinées à renforcer la vision du bon usage défendu par l'Académie, ces remarques sont proposées en complément des marques diastratiques :

\begin{abstract}
«Il nous est également apparu que nos notations habituelles : familier, populaire, vulgaire, argotique, trivial, avaient de moins en moins d'effet dissuasif, comme si, même assortis de ces mentions, le fait que des mots grossiers soient mentionnés " dans le dictionnaire " autorisait leur emploi sans discernement ni retenue. Que nous ayons dû en faire état, parce qu'ils sont d'un usage parlé, hélas fréquent, ne saurait constituer un encouragement à s'en servir en aucune occasion qui commande, oralement ou dans l'écrit, un langage correct.
\end{abstract}

Aussi avons-nous introduit de place en place des remarques normatives, bien visibles, qui proscrivent les expressions, constructions ou utilisations le plus agressivement fautives et dont on peut craindre qu'elles ne s'installent dans le mauvais usage. » (Avertissement, 1992)

Contestant l'efficacité dissuasive des marques de registres traditionnelles, les académiciens ont introduit les remarques normatives dans le but de proscrire "les expressions, constructions ou utilisations le plus agressivement fautives et dont on peut craindre qu'elles ne s'installent dans le mauvais usage"8. Résolument normatives, ces remarques permettent de mieux éclairer le rôle également normatif attribué aux marques de registres au sein de ce dictionnaire du bon usage.

La lecture de l'ouvrage de J.-P. Caput (1986) nous informe que la mise en place du dispositif des remarques normatives ne constitue pas un phénomène totalement nouveau. Certaines recommandations linguistiques ont effectivement fait l'objet de publications sous la forme de feuillets séparés du dictionnaire. J.-P. Caput évoque avec précision l'existence de ces « Mises en garde » :

\begin{abstract}
«Cette dénomination aux apparences menaçantes cache tout simplement des conseils que l'Académie, comme c'est sa mission, donne au public sur le plan linguistique. Sans que soit précisé à qui elles s'adressent plutôt, elles valent, sinon toutes du moins telle ou telle partie d'entre elles, pour un peu tout le monde. D'ailleurs, les ouvrages ou les simples opuscules, fort nombreux, qui vont dans le même sens se vendent très bien en librairie.

Il s'agit de quelques feuillets, actuellement encore ronéotypés, consignant des remarques placées dans l'ordre alphabétique et se présentant ainsi : en marge, le terme ou les termes, la tournure grammaticale incriminés; en face, un bref développement expliquant en quoi consiste l'erreur, apportant un ou plusieurs exemples et proposant souvent plusieurs solutions pour éviter l'écueil. La périodicité de ces Mises en garde est variable, mais en général, il en paraît au moins une par an. Malheureusement, leur diffusion est encore restreinte; sans doute le fait qu'à partir de décembre 1985 elles soient imprimées leur permettra d'obtenir une plus large diffusion dans le public. »
\end{abstract}

J.-P. Caput poursuit plus loin la présentation de ces remarques : 
« Afin de donner un exemple à la fois concret et récent, nous allons étudier celles du 7 février et du 13 juin 1985. On peut ranger ces 62 remarques sous quatre rubriques dont chacune admet des subdivisions; [...]

Problèmes grammaticaux $[\ldots]$

Vocabulaire [...]

Registres [...]

Orthoépie (prononciation correcte) [...]

Cette liste appelle quelques commentaires. D'abord sur le plan quantitatif, on peut constater l'importance privilégiée accordée au vocabulaire : 37 remarques sur un total de 62 - près des deux tiers; dans cette rubrique, ce qui bénéficie de la plus grande attention, c'est, dans l'ordre : les anglicismes (11 attestations, dont 6 concernant des mots anglais ou américains); viennent ensuite les problèmes de sens : en général (10), mais ceux-ci prédominent si on les regroupe (13 en tout); enfin, les néologismes préoccupent l'Académie : 7 cas. La grammaire vient en deuxième place, avec 11 remarques; il est intéressant de noter que près de la moitié se rapportent à des faits de syntaxe. Enfin, outre une rectification portant sur la prononciation du mot "antienne", nous trouvons un souci tout à fait justifié actuellement pour les registres de langue et les registres d'emploi; si ces derniers mettent en cause l'intrusion d'un vocabulaire technique ou scientifique spécialisé dans la langue commune, les premiers ne sont autres que ce que l'on appelait autrefois "les niveaux de langue. » (populaire, familier, etc.) [...] (Caput, $1986: 93)$

Les statistiques de J.-P. Caput sur la répartition par grands domaines des remarques proposées dans les feuillets nous semblent tout à fait intéressantes et mériteraient - chose que nous ne pourrons faire ici faute de temps - d'être examinées en fonction du corpus que nous avons établi pour la présente étude.

Parues depuis un certain nombre d'années sous la forme des feuillets décrits ci-dessus, les remarques normatives livrées par l'Académie ne constituent donc pas un phénomène nouveau mais un phénomène relativement méconnu :

«En réalité, le problème réside dans la diffusion; peu de personnes, proportionnellement, connaissent l'existence - a fortiori le contenu - de ces feuillets. Peut-être la presse écrite pourrait-elle jouer un rôle important, sinon déterminant, pour faire connaître ces Mises en garde. » (Caput, 1986 : 93)

La méconnaissance des feuillets normatifs semble en grande partie imputable au(x) mode(s) de diffusion de ces derniers et aux technologies sur lesquelles s'est appuyée cette diffusion. La mise en ligne récente sur le site internet de la Compagnie - de ces recommandations constitue une occasion de leur donner une meilleure lisibilité, voire une toute première lisibilité.

En dehors de ce facteur de mauvaise diffusion dont ont pu souffrir les feuillets normatifs, la séparation physique de ces derniers d'avec le dictionnaire lui-même pourrait constituer l'explication la plus probante de leur méconnaissance. Ainsi qu'en atteste l'existence même de notre communication ${ }^{9}$, l'intégration des remarques au sein même des articles de la neuvième édition du $D A F$ semble avoir réglé - au moins en partie - ce problème de visibilité.

C'est en effet précisément l'intégration de ces remarques à la nomenclature du dictionnaire qui constitue la véritable nouveauté de la neuvième édition du $D A F$ et qui de fait lui confère un caractère davantage normatif.

\subsection{Une ouverture à la variation linguistique}

Un autre dispositif lexicographique identifié comme une innovation dans cette dernière édition est l'introduction de nombreux marquages régionalisants qui dans l'absolu semblent caractériser une plus grande prise en compte de la variation linguistique. Ces marquages renvoient en fait à une pluralité de 
séquences comprenant certes la forme « régional(e)» mais aussi la forme « dialectal » et les mentions de noms de variétés linguistiques régionales hexagonales (« picard», « normand», «provençal», « breton », " gallo », " gascon», " alsacien », etc.) ou extra-hexagonales (" suisse »), voire certaines périphrases du type «se dit en/à ». C'est pour cette raison que nous utilisons ici l'hyperonyme "régionalisant », dans la mesure où ce type de marques, plurielles, n'est pas stabilisé.

Dans la préface de la neuvième édition de leur dictionnaire, les académiciens évoquent l'introduction de ces régionalismes :

\begin{abstract}
« D'autre part, elle [l'Académie] s'est gardée d'exclure certains mots d'emploi régional, et elle a donné accueil à des vocables tantôt conservés et tantôt inventés dans divers pays du vaste espace francophone, considérant qu'ils étaient de nature à enrichir la langue commune. » (Préface de la neuvième édition du $D A F$ )
\end{abstract}

L'analyse du mécanisme d'introduction de ces marquages régionalisants constitue ainsi une occasion de vérifier si la notion de « bon usage » si chère aux académiciens évolue dans la dernière édition du $D A F$.

L'introduction d'un certain nombres d'entrées proprement régionales ou d'un marquage régionalisant dans des articles déjà existants semble confirmer cette hypothèse. Les articles CAGOUILLE et MAGNAN illustrent tout d'abord le phénomène d'introduction de nouvelles entrées :

«*CAGOUILLE n. f. XVIIe siècle. Issu du latin populaire conchylia, « coquille ».

Nom dialectal de l'escargot. La pluie a fait sortir les cagouilles. » (Neuvième édition du $D A F)$

«*MAGNAN n. m. XVIII ${ }^{\mathrm{e}}$ siècle. Mot provençal.

En Provence, ver à soie. » (Neuvième édition du $D A F$ )

Les articles AGACE et CALOT illustrent quant à eux le phénomène d'introduction d'un marquage spécifiant la dimension régionale des mots définis :

«AGACE. n. f. Oiseau qu'on nomme plus communément Pie. Quelques-uns écrivent AGASSE. » (Huitième édition du $D A F$ )

«AGACE n. f. XIe siècle, agace ; XVIe siècle, agasse. Emprunté de l'ancien provençal agassa, lui-même de l'ancien haut allemand agaza, dérivé de aga, « pie ». Nom dialectal de la pie (on écrit aussi Agasse). » (Neuvième édition du $D A F$ )

«CALOT. n. m. Coiffure des soldats en petite tenue appelée auparavant Bonnet de police. » (Huitième édition du $D A F$ )

«I. CALOT n. m. XIXe siècle. Dérivé de cale, " sorte de bonnet », mot dialectal du Nord-Est de la France, d'origine obscure. Coiffure militaire légère, sans bord ni visière, de forme étroite et allongée. Coiffure civile de même forme. Porter le calot sur l'oreille.

II. CALOT n. m. XIX ${ }^{\mathrm{e}}$ siècle. Emploi métaphorique de calot, « noix », terme dialectal, dérivé du moyen français cale, de même sens.

Grosse bille dont les enfants se servent pour jouer. » » (Neuvième édition du $D A F$ )

À travers ces deux types d'exemples, il semblerait en effet que la dernière édition du répertoire des Académiciens manifeste une évolution conséquente avec la prise en compte de la problématique régionale et le fait en particulier d'introduire des articles nouveaux qui ne sont présentés qu'à titre de régionalismes. Néanmoins, globalement les modalités de mise en œuvre de ces marquages viennent nuancer ce qui pourrait être vu comme un progressisme de l'Académie. En effet la prise en compte des langues régionales passe surtout - ainsi que nous le montrerons ci-dessous - par la généralisation des 
informations étymologiques. L'article AFFIQUET reproduit ci-dessous fournit une illustration de ce qui ne constitue en fait qu'une indication étymologique :

«AFFIQUET n. m. XIIe siècle. Terme normanno-picard. Diminutif d'affique, forme normanno-picarde de l'ancien français affiche, « attache, boucle».

1. Vieilli. Surtout au pluriel. Objet de parure, colifichet. Porter des affiquets. 2. Petit capuchon qu'on ajuste à la pointe des aiguilles à tricoter quand on interrompt son ouvrage, pour empêcher les mailles de glisser. » ( Neuvième édition du $D A F$ )

En d'autres termes, le «bon usage » des académiciens n'a dans les faits pas encore pleinement évolué vers un «bon usage régional », en dépit du discours tenu sur leurs propres pratiques lexicologiques par les Académiciens ${ }^{10}$.

\section{Un ouvrage repensé}

En plus des innovations que nous venons de mentionner, la dernière édition du $D A F$ se distingue également par des choix lexicographiques qui dans une certaine mesure déconstruisent plusieurs positionnements qui avaient jusqu'à présent fait l'originalité du répertoire. Avec l'introduction de l'étymologie - que nous venons d'évoquer - et la mention d'écrivains célèbres, les Académiciens livrent ainsi un répertoire fortement repensé.

\subsection{Introduction de l'étymologie}

La première innovation significative qu'introduit cette dernière édition du $D A F$ concerne l'étymologie. Alors qu'ils s'étaient jusqu'alors toujours opposés à son marquage, les Académiciens reviennent sur leur jugement :

«En un temps, le nôtre, où, de toute évidence, la connaissance du latin et du grec se raréfie, parce qu'ils sont, hélas, de moins en moins enseignés, il nous est apparu d'un réel intérêt de rappeler, fût-ce brièvement, que la plupart de nos vocables plongeaient leurs racines, profondément, dans ces deux langues anciennes.

Aussi, pour la première fois dans l'histoire du Dictionnaire de l'Académie, c'est-à-dire depuis trois siècles, la définition de chaque nom ou terme sera précédée d'indications étymologiques. Indications volontairement succinctes, et qui ne prétendent pas à l'érudition, afin de se garder d'aucune pédanterie, mais qui permettront, en esquissant l'histoire des mots et leur évolution sémantique, voire orthographique, de mieux éclaircir leur sens et leur usage présent. » (Avertissement de 1992)

Ce passage positionne la question du marquage étymologique du lexique comme une préoccupation désormais importante du répertoire. Un examen approfondi de l'introduction de ce marquage étymologique montre toutefois que même si seules les langues latine et grecque sont mises en avant, ce sont en réalité une multiplicité de langues qui se retrouvent sur le devant de la scène linguistique grâce à l'introduction de ce nouveau dispositif lexicographique. Les articles BANDOULIĖRE, GOÉMON et PELOTARI illustrent ainsi respectivement les mentions du Catalan, du Breton et du Basque :

«BANDOULIÈRE n. f. XVIe siècle. Emprunté du catalan bandolera, dérivé de bandoler, « hors-la-loi ».

Bande de cuir ou d'étoffe, passée comme une écharpe d'une épaule à la hanche opposée pour porter un objet. Le fusil en bandoulière. Un sac, une gibecière, un cartable en bandoulière. »

« GOÉMON n. m. XIVe siècle, goumon. Emprunté du bas breton gwemon, « varech».

Nom générique donné à diverses algues marines brunes qu'on recueille sur certains rivages pour en faire des engrais ou en extraire des éléments chimiques. Du fumier de goémon. (On dit aussi Varech.)» 
« PELOTARI n. m. XIXe siècle. Emprunté du basque pelotari, de même sens.

Joueur de pelote basque. »

Aux différentes variétés linguistiques de France viennent s'ajouter de nombreuses langues étrangères ayant constitué un fond lexical au sein duquel le français est venu puiser au fil du temps.

En revenant sur la position ferme qu'elle affichait depuis toujours, l'Académie introduit une évolution majeure qui offre incontestablement un visage nouveau à son dictionnaire.

\title{
4.2 Les citations d'auteurs
}

Une seconde évolution significative relevée dans la dernière édition du $D A F$ concerne le choix d'exemplification retenu par l'Académie. Alors que celle-ci s'était jusqu'alors toujours appuyée sur des exemples forgés, la dernière édition du $D A F$ intègre pour la première fois des renvois vers des ouvrages littéraires au sein desquels se trouvent illustrés les mots décrits en entrée. Dans la préface et l'Avertissement de cette nouvelle édition se trouve mentionnée l'introduction de ce nouveau dispositif :

\begin{abstract}
« Pour cette raison, le Dictionnaire, par tradition, ne comporte pas de citations, ni ne fait presque jamais référence nominale à des auteurs. Par discrétion aussi ; les citations, s'il y en avait, seraient par la force des choses empruntées, pour un grand nombre, à des membres disparus ou présents de la Compagnie. » (Préface 1986)

« Nous avons dans le corps des articles respecté notre tradition qui veut que nous ne fassions pas appel à des citations, et que nous forgions nous-mêmes nos exemples d'emplois et de constructions, en nous obligeant autant qu'il se peut à la simplicité. Toutefois, autre innovation, nous avons cru bon de mentionner, pour certains mots, des titres d'œuvres célèbres où ils apparaissent, et qui leur ont conféré une illustration. » (Avertissement de 1992)
\end{abstract}

Tout en se défendant explicitement d'avoir dérogé à la règle initiale qu'elle s'était fixée de ne pas avoir recours à des citations d'auteurs, l'Académie introduit là un mécanisme lexicographique qui marque lui aussi une rupture importante avec les éditions précédentes de son dictionnaire.

\footnotetext{
«Nous avons dans le corps des articles respecté notre tradition qui veut que nous ne fassions pas appel à des citations, et que nous forgions nous-mêmes nos exemples d'emplois et de constructions, en nous obligeant autant qu'il se peut à la simplicité. $[\ldots]$

Toutefois, autre innovation, nous avons cru bon de mentionner, pour certains mots, des titres d'œuvres célèbres où ils apparaissent, et qui leur ont conféré une illustration. » (Avertissement de 1992)
}

Dans son article de $2009^{11}$, Samuel Souffi révèle ainsi que ce sont pas moins de 543 articles (de ABSOLU à $\mathrm{PIE}$ ) et 137 articles (de AMOUR à PHYSIOLOGIE) qui mentionnent un titre d'œuvre célèbre dans cette dernière édition du $D A F$. Ces titres d'ouvrages illustrant l'emploi du mot défini se trouvent sobrement introduits par la mention «Titres célèbres » puis listés les uns après les autres avec les références concernant leur auteur et leur date de publication. Les articles CHAPERON, FOURMI, HARMONIE et PRINCESSE rendent compte de l'introduction de ce nouveau dispositif :

«CHAPERON [...] Titre célèbre : Le Petit Chaperon rouge, de Charles Perrault (1697). » (Neuvième édition du $D A F)$

«FOURMI [...] Titres célèbres : La Cigale et la Fourmi ; La Colombe et la Fourmi, fables de Jean de La Fontaine (publiées en 1668) ; La Vie des fourmis, de Maurice Maeterlinck (1930). » (Neuvième édition du $D A F$ )

«HARMONIE [...] Titres célèbres : Harmonies de la nature, de Bernardin de SaintPierre (1796); Harmonies poétiques et religieuses, de Lamartine (1830). » (Neuvième édition du $D A F)$ 
« PRINCESSE [...] Titres célèbres : La Princesse d'Élide, comédie-ballet de Molière (1664) ; La Princesse de Clèves, de Madame de La Fayette (1678); La Princesse de Babylone, conte philosophique de Voltaire (1768). » (Neuvième édition du DAF)

Une fois de plus, en proposant d'introduire ces titres célèbres, les Académiciens semblent avoir repensé leur ouvrage et quelque peu adapté leur discours. Même si ce dispositif n'équivaut tout de même pas à l'introduction de citations d'auteurs, il témoigne d'un infléchissement académique et d'une ouverture hors de l'excellence académique vers les grands auteurs de la littérature française et francophone.

Nous terminerons notre réflexion sur ce point en nous demandant si un tel changement, au même titre que l'introduction du marquage étymologique, ne traduit pas un accroissement de la place accordée à la dimension dictionnairique ${ }^{12}$ par la vénérable institution.

\subsection{Une plus grande prise en compte de la Francophonie}

Un dernier critère d'évolution particulièrement significatif de la neuvième édition du $D A F$ réside dans la volonté affichée par l'Académie de se «préoccuper» de manière «désormais permanente » de la Francophonie :

«Soulignons un dernier point. Préoccupation nouvelle, mais désormais permanente, la Francophonie, au long de nos travaux, est toujours présente à notre esprit. Quand nous examinons, acceptons, définissons, redéfinissons les mots, nous pensons à tous les peuples à travers la Terre qui ont la langue française en partage ; nous pensons à tous les pays qui en font usage pour tout ou partie de leurs communications et de leurs échanges ; nous pensons à ceux qui l'emploient pour rédiger leurs lois. Nous pensons aux hommes et aux femmes, par millions sans cesse croissants, qui lui confient l'expression de leurs cultures, donc un peu de leur âme. » (Avertissement, 1992)

Avouons-le bien volontiers, ce passage - de tonalité pour le moins optimiste - reste relativement flou quant aux modalités de prise en compte de la Francophonie dans cette dernière édition du dictionnaire. Cet intérêt se traduit-il par un changement des modalités définitoires ou d'exemplification des articles ? Se traduit-il par une introduction massive de mots issus de la Francophonie ?

Même si nous n'avons pas eu le temps d'examiner les éventuels changements en ce qui concerne les modalités définitoires ou d'exemplification, nous avons pu constater qu'en effet cette dernière édition du répertoire des Académiciens accordait une large place aux mots de la francophonie. Même non quantifié par faute de temps, cet accroissement des mots issus de la francophonie est manifeste et se trouve illustré par les quelques exemples reproduits ci-dessous, mettant en scène des lexèmes venus du Canada, de Suisse, d'Afrique du nord ou d'Afrique noire :

«*BLANCHON n. m. XXe siècle. Dérivé de blanc.

Se dit au Canada du petit du phoque, tant qu'il a sa fourrure blanche. » (Neuvième édition du $D A F$ )

«*BOUBOU n. m. XIXe siècle. Emprunté du malinké, langue de Guinée, bubu, « singe », « peau de singe ».

Longue tunique flottante portée en Afrique noire. » (Neuvième édition du $D A F$ )

«*BOURGEOISIAL, -ALE adj. (pl. Bourgeoisiaux, -ales). XVIIe siècle. Dérivé de bourgeois.

En Suisse. Relatif au bourgeois ou à la bourgeoisie ; administré par les bourgeois. Pâturages bourgeoisiaux. Vignes bourgeoisiales. » (Neuvième édition du DAF)

«*BRUNANTE n. f. XIXe siècle. Dérivé du radical de brunir.

Au Canada, se dit du déclin du jour. Il est venu à la brunante. » (Neuvième édition du $D A F)$

«*HAMBELLANIE n. f. XVIIe siècle. Dérivé de chambellan. 
(Maroc). Charge de chambellan ; bureau et services du chambellan. » (Neuvième édition du $D A F$ )

«*CHERGUI n. m. XXe siècle. Mot de l'arabe maghrébin, de l'arabe classique charqi, « oriental».

Au Maroc, nom du sirocco. » (Neuvième édition du $D A F$ )

«*GANDOURA n. f. XVIIIe siècle, gandoure. Emprunté de l'arabe gandura, de même sens.

Ample tunique, portée surtout en Afrique du Nord. » (Neuvième édition du $D A F$ )

Conformément à ce qui a été annoncé, les Académiciens semblent avoir considérablement ouvert leur répertoire aux mots de la francophonie, conférant ainsi à nouveau un visage différent à leur travail.

\title{
5 Une adaptation à la modernité
}

$\mathrm{Au}$ fil de ses éditions, le $D A F$ a connu un certain nombre d'évolutions scientifiques plus ou moins majeures. Au rang de celles-ci, citons par exemple le passage d'un classement des entrées lexicographiques par racines (édition de 1694) à un classement alphabétique (dès l'édition de 1718). Rappelons également l'intégration de changements orthographiques conséquents dans les éditions de 1740 et 1762, respectivement dirigées par Charles Duclos et Fabre d'Olivet, ou bien encore la publication d'un lexique de termes propres à la Révolution lors de l'édition de 1798.

À l'image de ces différentes éditions, la neuvième édition du $D A F$ apporte, nous l'avons vu, son lot d'évolutions et d'innovations importantes. Celle-ci concentre même un nombre significatif d'évolutions qui lui confèrent un aspect très différent des éditions précédentes.

Notre intérêt s'est ensuite porté sur des procédés qui tendent tous à faire du $D A F$ un dictionnaire plus accessible au grand public.

Ainsi que l'illustrent les nombreux projets de rétroconversion des dictionnaires anciens dont les éditions antérieures du $D A F$ ont d'ailleurs bénéficié (Wooldridge 1994 et Wooldridge et Leroy-Turcan 1996) ${ }^{13}$, la lexicographie est incontestablement entrée dans l'ère du numérique. La mise à disposition et la consultation de cette neuvième édition du $D A F$ sur Internet renforce ce constat :

\section{«L'Académie n'entend pas ignorer pour autant les nouvelles formes de diffusion qui permettent d'avoir, par le moyen de l'internet, un accès immédiat et aisé au Dictionnaire. » (Avant-propos de la neuvième édition)}

Tout en intégrant le dictionnaire dans la dynamique incontournable de l'informatisation, la mise en ligne du $D A F$ constitue pour l'Académie une occasion formidable de mieux faire connaître son ouvrage, mais aussi d'assurer son accès auprès d'un nombre de locuteurs bien plus important.

De la même façon, la mise sur le marché d'exemplaires du $D A F$ à un prix réduit - à peine une trentaine d'euros - et dans un format de poche très maniable ${ }^{14}$, semblent faire de ce dictionnaire un répertoire s'ouvrant désormais davantage au grand public ${ }^{15}$. C'est d'ailleurs la volonté explicitement affirmée par l'Académie à travers la voix de son secrétaire perpétuel Hélène Carrère d'Encausse :

\begin{abstract}
«En 1994, l'Académie française décidait, sans attendre l'achèvement de la nouvelle édition de son Dictionnaire, de mettre à la disposition du public, dans une présentation qui la rende accessible à tous, le premier tome, dont l'Imprimerie nationale avait assuré la publication en 1992. La presse s'est largement fait l'écho de cet évènement sans exemple dans l'histoire de notre Compagnie. » (Avant-propos de la neuvième édition du $D A F$ )
\end{abstract}

Le phénomène d'introduction des remarques normatives évoqué plus haut peut lui aussi être interprété comme une initiative supplémentaire en faveur de cette ouverture vers le grand public ${ }^{16}$. Par ce biais, l'Académie, qui propose un dictionnaire du bon usage et un dictionnaire de l'institution française, semble 
en effet user de toute sa légitimité pour proposer ce type de recommandations normatives et donc s'offrir une visibilité accrue auprès du public.

\section{Conclusion}

De manière certes quelque peu lapidaire, nous avons essayé de mettre en évidence à quel point la dernière édition du $D A F$ symbolisait un renouveau significatif du travail lexicographique dont bénéficie le dictionnaire de la vénérable institution.

Non seulement la neuvième édition de ce répertoire propose un certain nombre d'innovations lexicographiques importantes, mais en plus traduit-elle une réflexion des Académiciens qui semble aller dans le sens d'une inflexion de certaines positions caractéristiques du travail académique depuis quatre siècles. L'introduction du marquage étymologique et la place accordée aux titres d'œuvres célèbres - pour ne citer que ces phénomènes - raisonnent à ce titre comme des manifestations d'un changement de la pensée des Immortels.

Un tel renouveau suscite incontestablement de nombreuses interrogations mais traduit peut-être simplement les affres que même l'Académie Française connaît face aux mutations de l'objet dictionnaire depuis la fin du $\mathrm{XX}^{\mathrm{e}}$ siècle. La mise en ligne sur Internet et la diffusion de l'ouvrage dans un format plus commode et à bas prix illustrent peut-être le poids toujours plus grand de la dictionnairique dans les processus lexicographiques mêmes.

\section{Références bibliographiques}

Académie française, Dictionnaire de l'Académie française - Tome 1 A-Enz et Tome 2 Eoc-Map (9e édition). (2005). Paris, Fayard.

CAPUT, J-P. (1986). L'Académie française, Collection «Que sais-je ?», Paris, PUF.

Le Dictionnaire de l'Académie française : histoire et nuances de la langue française (1694-1935). (2000). Éditions Redon.

Le Dictionnaire de l'Académie française (9 édition). http://atilf.atilf.fr/academie9.htm

PRUVOST, J. (2005). «Quelques concepts lexicographiques opératoires à promouvoir au seuil du XXIe siècle », ÉLA, $\mathrm{n}^{\circ} 137$, pp. 7-37.

QUEMADA, B. et al. (1997). Les Préfaces du Dictionnaire de l'Académie française (1694-1992): textes, introductions et notes. Paris, Champion.

QUEMADA, B., PRUVOST, J. (1998). Le Dictionnaire de l'Académie française et la lexicographie institutionnelle européenne. Paris : Honoré Champion.

REY, C. (2012a). «Les 'Recommandations normatives' de la neuvième édition du Dictionnaire de l'Académie Française : une lexicographie institutionnelle assumée ", Carnets d'Atelier de Sociolinguistique, Éditions 1'Harmattan, pp. 59-83.

REY, C. (dir.). (2012b). Etudes de Linguistique Appliquée (ÉLA) n 163, «L'Académie Française : un modèle qui traverse les siècles », Éditions Klincksieck.

REY, C., PIEROZAK, I. (À paraître). « Du « bon usage régional » dans la Neuvième édition du Dictionnaire de l'Académie Française : regards sociolexicologiques croisés", Colloque "Bon Usage et Variation Sociolinguistique: Perspectives diachroniques et traditions nationales „), 16-18 juillet 2009, Murray Edwards College, Université de Cambridge.

REY, C. (2010). "Éléments de réflexion concernant les processus lexicographiques, lexicologiques et métalexicographiques à l'ère d'internet ", in Cahiers de linguistique, 36/2, Du " terrain " à la relation : expériences de l'internet et questionnements méthodologiques (Sous la direction d'Isabelle Pierozak), Presses Universitaires de Rennes, pp. 71-80.

SOUFFI, S. (2009). «Le Dictionnaire de l'académie française : entre bon usage et culture », Ela. Études de linguistique appliquée., 2009/2 n 154, pp. 155-176. 
WOOLDRIDGE, T.R., LEROY-TURCAN, I. (1996). « Les mots-clefs métalinguistiques comme outil d'interrogation structurante des dictionnaires anciens ", Lexicomatique et dictionnairiques (éd. A. Clas, P. Thoiron \& H. Béjoint), Beyrouth: FMA \& Montréal: AUPELF-UREF, pp. 307-16.

WOOLDRIDGE, T.R. (1994). «Projet d'informatisation du Dictionnaire de l'Académie (1694-1935)», Actes du Colloque international Le Dictionnaire de l'Académie française et la lexicographie institutionnelle européenne, Institut de France, novembre 1994; (ed. B. Quemada \& J. Pruvost), Paris, Champion, pp. 309-20. 
${ }^{1}$ Exemple : Les évolutions de l'orthographe.

${ }^{2}$ Exemple : Intégration dans l'édition de 1798 de nombreux mots liés à la Révolution Française.

${ }^{3}$ Cf. également QUEMADA, 1998.

${ }^{4}$ REY, Christophe (dir.), (2012b). Études de Linguistique Appliquée (ÉLA) n 163, «L'Académie Française: un modèle qui traverse les siècles », Éditions Klincksieck.

${ }^{5} \grave{A}$ ce jour sont disponibles les articles allant de $A$ à Quadrivium.

${ }^{6}$ http://www.journal-officiel.gouv.fr/dae.html

${ }^{7}$ Nous ne parlerons pas ici de la réorganisation sémantique des articles évoquées dans l'Avertissement, ni de la prise en compte massive de termes techniques (Médecine, Informatique, Communications, etc.).

${ }^{8}$ Avertissement, 1992.

${ }^{9} \mathrm{Cf}$. également REY (2012a).

${ }^{10} \mathrm{Cf}$. REY et PIEROZAK, (À paraître).

${ }^{11}$ SOUFFI, S., 2009, « Le Dictionnaire de l'académie française : entre bon usage et culture », Ela. Études de linguistique appliquée., $2009 / 2 n^{\circ} 154$, p. $155-176$.

${ }^{12} \mathrm{Cf}$. PRUVOST, J. 2005. «Quelques concepts lexicographiques opératoires à promouvoir au seuil du XXIe siècle », ÉLA, n $137, \mathrm{p}$. 7-37.

${ }^{13}$ Les éditions Redon ont d'ailleurs proposé un CD-ROM sur lequel figurent les huit premières éditions du $D A F$.

${ }^{14}$ Publication chez Fayard en 2005.

${ }^{15}$ Cf. Rey, (2010)

${ }^{16}$ Nous n'excluons néanmoins pas de notre positionnement l'idée soulevée par Jean Pruvost, idée selon laquelle la question de la personnalité des académiciens - en particulier de celle du Secrétaire Perpétuel - peut aussi avoir une incidence dans la mise en place de choix tels que celui. 